\title{
Prefrontal Control of the Amygdala
}

\author{
Ekaterina Likhtik, Joe Guillaume Pelletier, Rony Paz, and Denis Paré \\ Center for Molecular and Behavioral Neuroscience, Rutgers, The State University of New Jersey, Newark, New Jersey 07102
}

Accumulating evidence indicates that phobic and posttraumatic anxiety disorders likely result from a failure to extinguish fear memories. Extinction normally depends on a new learning that competes with the original fear memory and is driven by medial prefrontal cortex (mPFC) projections to the amygdala. Although mPFC stimulation was reported to inhibit the central medial (CEm) amygdala neurons that mediate fear responses via their brainstem and hypothalamic projections, it is unclear how this inhibition is generated. Because the mPFC has very sparse projections to CEm output neurons, the mPFC-evoked inhibition of the CEm is likely indirect. Thus, this study tested whether it resulted from a feedforward inhibition of basolateral amygdala (BLA) neurons that normally relay sensory inputs to the CEm. However, our results indicate that $\mathrm{MPFC}$ inputs excite rather than inhibit BLA neurons, implying that the inhibition of CEm cells is mediated by an active gating mechanism downstream of the BLA.

Key words: amygdala; medial prefrontal cortex; infralimbic; prelimbic; extinction; fear conditioning

\section{Introduction}

Congruent findings from studies of fear learning in animals and anxiety disorders in humans indicate that research on the circuits mediating fear acquisition constitutes our best hope of understanding anxiety disorders. The experimental paradigm typically used to study this process is pavlovian fear conditioning, a form of learning that depends on the amygdala in animals (LeDoux, 2000; Maren, 2001) and humans (Bechara et al., 1995; LaBar et al., 1995). However, more important from a clinical perspective is to understand how fear responses subside. Experimentally, this is modeled with repetitive presentations of the conditioned stimulus (CS), resulting in the decline of conditioned fear to preconditioning levels, a process termed extinction (Pavlov, 1927; Myers and Davis, 2002).

Much data implicate the medial prefrontal cortex (mPFC) in extinction. mPFC lesions (Morgan and LeDoux, 1995; Quirk et al., 2000) and inhibition of protein synthesis in the mPFC (Santini et al., 2004) block extinction recall. Moreover, functional imaging studies indicate that the $\mathrm{mPFC}$ is engaged during extinction (Phelps et al., 2004) and that subjects with posttraumatic stress disorder (PTSD) have reduced $\mathrm{mPFC}$ activity during trauma recall (Bremner et al., 1999). Finally, mPFC stimulation inhibits conditioned fear (Milad and Quirk, 2002).

Similarly, much data implicate the amygdala in extinction. Infusions of NMDA antagonists in the amygdala disrupt extinction (Falls et al., 1992), whereas drugs facilitating NMDA responses accelerate extinction in animals (Walker et al., 2002) and phobic patients (Ressler et al., 2004). Moreover, although CS-

Received March 9, 2005; revised July 1, 2005; accepted July 1, 2005.

This work was supported by National Institutes of Health Grants R01 MH-066856-01 and R01 MH-073610-01. We thank Drs. G. J. Quirk and J. M. Tepper as well as members of the Paré laboratory for comments on a previous version of this manuscript.

Correspondence should be addressed to Denis Paré, Center for Molecular and Behavioral Neuroscience, Rutgers, The State University of New Jersey, 197 University Avenue, Newark, NJ 07102. E-mail: pare@axon.rutgers.edu. D01:10.1523/JNEUROSCI.2314-05.2005

Copyright $\odot 2005$ Society for Neuroscience $\quad 0270-6474 / 05 / 257429-09 \$ 15.00 / 0$ evoked responses of some basolateral amygdala (BLA) neurons decrease during extinction (Quirk et al., 1995; Hobin et al., 2003), many BLA cells remain CS responsive (Repa et al., 2001). These observations, coupled with the fact that $\mathrm{MPFC}$ neurons display CS-evoked responses during extinction recall (Milad and Quirk, 2002), suggest that mPFC-amygdala interactions play a key role in extinction.

How might the mPFC participate in extinction? Given that amygdala outputs to structures mediating fear mostly originate in its central medial nucleus (CEm) (Holstege et al., 1996), this site may be an effective locus for mPFC control of conditioned fear. Consistent with this, $\mathrm{MPFC}$ stimulation inhibits CEm output neurons and central lateral neurons (Quirk et al., 2003). However, the mechanism of this mPFC-evoked inhibition remains unknown.

Because the mPFC sends only sparse projections to the CEm (McDonald, 1998), its inhibitory influence over the CEm is likely indirect. One possibility is that the $\mathrm{MPFC}$ acts via BLA neurons that normally relay sensory inputs to the CEm. Indeed, the $\mathrm{mPFC}$ sends a robust projection to the BLA (Russchen, 1982; Sesack et al., 1989; McDonald, 1998). However, mPFC axons ending in the BLA use glutamate as a transmitter, usually forming asymmetric synapses with principal cells but also with GABAergic interneurons (Brinley-Reed et al., 1995; Smith et al., 2000). Thus, mPFC stimulation should excite principal BLA neurons and lead to the excitation of BLA projection sites, such as the CEm (Krettek and Price, 1978). However, the observation that mPFC stimulation inhibits rather than excites CEm cells (Quirk et al., 2003) raises the possibility that $\mathrm{MPFC}$ inputs could also activate BLA interneurons, inhibiting BLA projection cells, thereby causing a disfacilitation of CEm neurons. This was tested using extracellular recordings of $\mathrm{mPFC}$ and BLA neurons in vivo.

\section{Materials and Methods}

All procedures were approved by the Institutional Animal Care and Use Committee of Rutgers State University in compliance with the Guide for the Care and Use of Laboratory Animals. Adult cats (2.5-3.5 kg) were 
preanesthetized with a mixture of ketamine and xylazine (15 and $2 \mathrm{mg} /$ $\mathrm{kg}$, i.m.) and artificially ventilated with a mixture of ambient air, oxygen, and isoflurane. Atropine sulfate $(0.05 \mathrm{mg} / \mathrm{kg}, \mathrm{i} . \mathrm{m}$.) was administered to prevent secretions. The end-tidal concentration in $\mathrm{CO}_{2}$ was kept at $3.7 \pm$ $0.2 \%$, and the body temperature was maintained at $37-38^{\circ} \mathrm{C}$ with a heating pad. The level of anesthesia was assessed continuously by monitoring the electroencephalogram (EEG) and electrocardiogram.

In the following, the BLA refers to a complex of three amygdala nuclei that includes the lateral, basomedial, and basolateral (BL) nuclei. Separate in vivo extracellular recordings were conducted in the BL nucleus of the amygdala (16 animals), the mPFC ( 3 animals), and at both sites simultaneously (4 animals). In the mPFC recording experiments, concentric stimulating electrodes were positioned in the BL nucleus bilaterally to antidromically activate mPFC neurons and infer their conduction velocity. In the BL recording experiments, we compared the type of responses evoked by electrical stimulation of various cortical areas. To this end, concentric stimulating electrodes $(0.7 \mathrm{~mm}$ spacing between tip and ring) were inserted in the ipsilateral and contralateral mPFC, the insula, as well as the temporal neocortex immediately lateral to perirhinal area 36. To position our mPFC stimulating electrodes in superficial layers, we used the electrode to monitor the EEG activity as it was lowered toward the $\mathrm{mPFC}$, using a near horizontal lateromedial approach. Previous studies have shown that many anesthetics induce a ubiquitous slow (0.5-1 $\mathrm{Hz}$ ) EEG oscillation in the cerebral cortex (Steriade et al., 1993; Collins et al., 2001). This oscillation is composed of a depth-negative-surfacepositive component associated to an increased gamma power, and a depth-positive-surface-negative component during which fast EEG rhythms are reduced (Steriade et al., 1993; Collins et al., 2001). Consistent with this, when the concentric electrode approached the deep mPFC layers, the spontaneous activity recorded by the tip and ring had the same polarity. Eventually, as the electrode was advanced further, the polarity of the slow oscillation recorded by the tip became opposite to that recorded by the ring. Histological controls consistently revealed that in this position, the tip of the stimulating electrode was in layer I.

In the same experiments, bipolar stimulating electrodes were also inserted in one of the main sources of inputs to the mPFC, the mediodorsal thalamic nucleus (MD) (Martinez-Moreno et al., 1987; Musil and Olson, 1991) as well as the contralateral mPFC (McDonald et al., 1996; Vertes, 2004), to study how indirect activation of the mPFC affects BL neurons. Here, it should be pointed out that, in this case, we aimed our stimulating electrodes to the infralimbic cortex to avoid activation of the prelimbic projections to the contralateral amygdala (McDonald et al., 1996; Vertes, 2004). In the simultaneous $\mathrm{BL}$ and $\mathrm{mPFC}$ recording experiments, arrays of high-impedance tungsten microelectrodes ( 8 and 12 , respectively) were used to correlate variations in spontaneous unit activity. In all experiments, the bone overlying the regions of interest was removed, and the dura mater was opened.

Lidocaine microinfusion. To test whether the MD-evoked BL responses were mediated by the $\mathrm{MPFC}$, lidocaine was infused in the $\mathrm{mPFC}$. A guide cannula ( $0.7 \mathrm{~mm}$ outer diameter) was inserted $1 \mathrm{~mm}$ rostral to the $\mathrm{mPFC}$ stimulating electrode ipsilateral to the BLA recording site. After locating a suitable BL test site, a microinfusion probe $(0.5 \mathrm{~mm}$ outer diameter $)$ was inserted through the guide cannula, and lidocaine (10\% in $0.9 \%$ saline solution) was passed through the probe (Boehnke and Rasmusson, 2001) at a rate of $0.2 \mu \mathrm{l} / \mathrm{min}$ using a micropump. Lidocaine was infused for a minimum of $20 \mathrm{~min}$ and a maximum of $40 \mathrm{~min}$. To assess the effects of lidocaine infusion, spontaneous EEG activity and MD-evoked field potentials were monitored in the $\mathrm{mPFC}$.

Extracellular recordings in the $m P F C$. The $\mathrm{MPFC}$ recording electrodes were aimed toward the prelimbic-infralimbic region using the same near-horizontal, lateromedial approach as with the mPFC stimulating electrodes described above. During the experiments, the electrodes were advanced in steps of $5 \mu \mathrm{m}$ by a micromanipulator and BL-evoked responses were recorded. Orthodromic responses appeared first, in keeping with a BL projection to both deep and superficial layers of the mPFC (Krettek and Price, 1977). As the recording electrodes approached the $\mathrm{mPFC}$ output layers to the BL nucleus (layers II-III), neurons generating antidromic responses were encountered. The electrodes were then ad- vanced into the contralateral $\mathrm{mPFC}$, and the experiment was repeated in the opposite hemisphere in three different animals.

Extracellular recordings in the BL. The spontaneous and evoked activity of BLA neurons was monitored by two to eight high-impedance tungsten microelectrodes (1 mm spacing) aimed at the BL nucleus. As the recording electrodes were advanced in steps of $5 \mu \mathrm{m}$, the various stimulation sites were tested $(0.05-0.1 \mathrm{~ms} ; 1 \mathrm{~mA} ; 0.5 \mathrm{~Hz})$. Consistent with a strong projection from the BLA to the ipsilateral mPFC (Krettek and Price, 1977; McDonald, 1991), once the microelectrodes reached the BL, mPFC stimuli evoked antidromic spikes in a large number of cells (see Results). After isolating responsive cells with a high signal-to-noise ratio $(\geq 3)$, the stimulus intensity was decreased just above threshold, and the type of responses evoked from each stimulation site (orthodromic vs antidromic) plus their latencies were noted. Threshold was defined differently for orthodromic versus antidromic responses. For orthodromic responses, threshold was defined as a stimulus intensity that synaptically activated the cell in $30-70 \%$ of trials. Because antidromic responses behaved in an all-or-none manner when the stimulation intensity was reduced, threshold was defined as the minimal stimulation intensity required to reliably backfire the recorded cell.

To be classified as antidromic, responses had to meet at least two of the following three criteria (Lipski, 1981) (see Fig. 3): (1) stable latency $(<0.3$ $\mathrm{ms}$ jitter), (2) collision with orthodromically evoked or spontaneously occurring spikes, and (3) response after high-frequency stimulation (300 $\mathrm{Hz}$ ). The spontaneous and evoked activity of responsive neurons was observed on a digital oscilloscope, digitized at $20 \mathrm{kHz}$, and stored on disk for off-line analysis.

Histological identification of recording and stimulating sites. At the end of the experiments, the animals were given an overdose of sodium pentobarbital $(50 \mathrm{mg} / \mathrm{kg}$, i.v.), and extracellular recording sites were marked with electrolytic lesions ( $0.6 \mathrm{~mA}$ for $10 \mathrm{~s})$. The brains were then taken out, fixed in $2 \%$ paraformaldehyde and $1 \%$ glutaraldehyde, sectioned on a vibrating microtome (at $100 \mu \mathrm{m}$ ), and stained with neutral red to reveal electrode placements. The microelectrode tracks were reconstructed by combining the micrometer readings and histology. To be included in the analysis, cells had to be histologically confirmed as being located in the $\mathrm{BL}$ nucleus or the mPFC.

Analysis. Analyses were performed off-line with commercial software (IGOR; WaveMetrics, Lake Oswego, OR) and custom-designed software running on Macintosh computers. Spikes were detected after digital filtering $(0.3-20 \mathrm{kHz})$ of the raw waves. Peristimulus histograms of unit discharges were computed. All values are expressed as means \pm SE.

\section{Results}

\section{Database}

A total of 539 neurons were recorded in this study: 140 in the mPFC region and 399 in the amygdala or immediately surrounding structures. However, the present study includes only cells that were histologically determined to be located in the prelimbic/ infralimbic areas of the mPFC $(n=124)$ or in the BLA $(n=359)$. It should be noted that because GABA interneurons account for a small proportion of the total neuronal population in the $\mathrm{mPFC}$ (Gabbott and Bacon, 1996) and BLA (McDonald and Mascagni, 2001), by chance alone, our samples should be mostly composed of projection cells.

\section{Correlation between the activity of MPFC and BLA neurons}

If $\mathrm{mPFC}$ neurons cause feedforward inhibition of principal BLA cells, then spontaneous firing of mPFC neurons should generally be correlated with a decreased firing probability in the BLA. To test this idea, we obtained simultaneous recordings of spontaneously active $\operatorname{mPFC}(n=76)$ and BLA $(n=65)$ neurons in four cats. Spikes generated by different cells were discriminated from each other and background noise using a clustering procedure and visual inspection of spike waveforms (Fig. 1A1,A2). Crosscorrelations of unit activity were computed for all mPFC-BLA cell couples $(n=587)$ using mPFC neurons as references. Indi- 

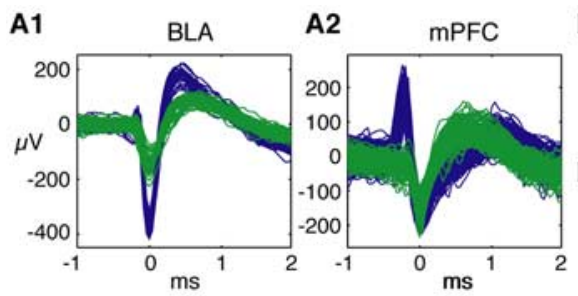

B1

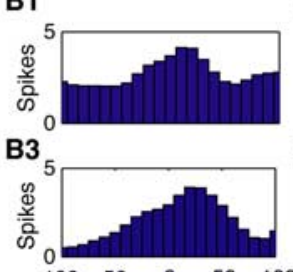

B2

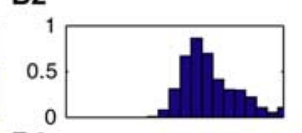

B4

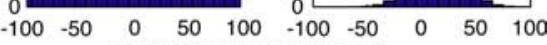

C z-score

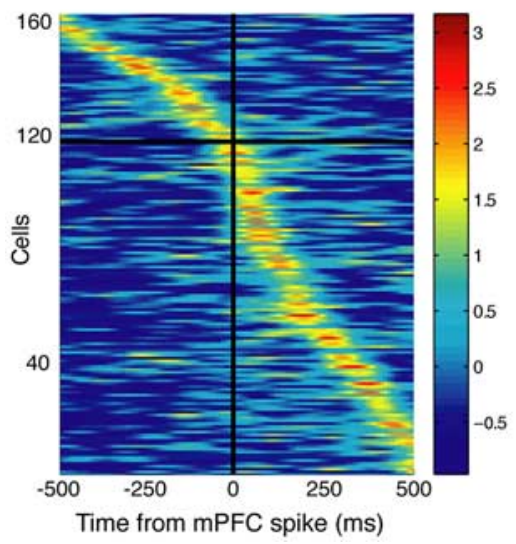

D1
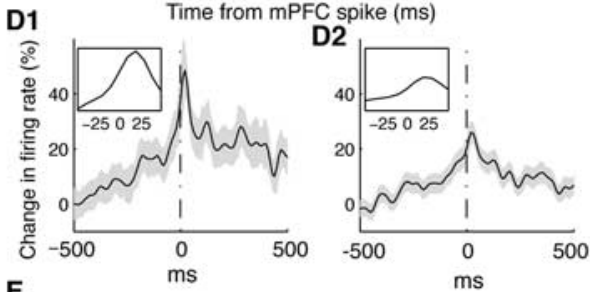

E

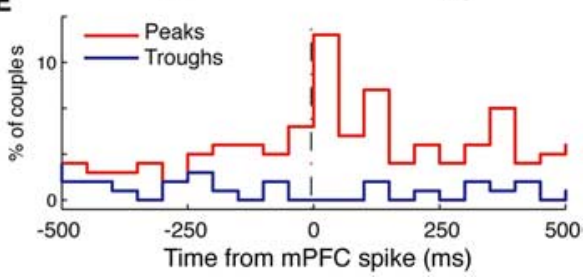

Figure 1. Spontaneous $\mathrm{mPFC}$ firing is associated with an increased firing probability in BL neurons. The activity of simultaneously recorded BLA and mPFC neurons was cross-correlated using mPFC cells as references. $\boldsymbol{A 1}, \mathbf{A 2}$, Waveforms of all spikes from different cells (blue and green traces) recorded by an electrode located in the BLA (A1) and an electrode located in the MPFC (A2). Cells were isolated off-line using a clustering algorithm. B1-B4, Examples of individual CCs obtained in different animals. These CCs peaked at $\sim 20-50 \mathrm{~ms}$ after the $\mathrm{mPFC}$ reference spike. C, Graph depicting all statistically significant mPFC $-B L C C$, sorted by their peak time. Bins of $10 \mathrm{~ms}$ are expressed in $z$-scores and presented in pseudocolor. To test for significance, we compared each bin in the $\pm 500 \mathrm{~ms}$ range to a Poisson distribution with mean $(\lambda)$ calculated from a baseline period ( -1000 to $-750 \mathrm{~ms})$. The $p$ value was Bonferroni-corrected for multiple bin comparisons, accounting for $-500 \mathrm{~ms}$ in $10 \mathrm{~ms}$ bins (i.e., $0.05 / 100)$. D1, D2, Average of all significant $\mathrm{mPFC}-\mathrm{BL} C \mathrm{CS}_{(}(\boldsymbol{D} 1)$ and of all available CCs (D2). The shaded area shows \pm SEM. Insets show expanded view of the histogram peaks. Note peak at $\sim 30 \mathrm{~ms}$. $\boldsymbol{E}$, Frequency distribution of the position of $C($ peaks (red) and troughs (blue) for the data shown in C (bins of $50 \mathrm{~ms}$ ). All depicted CCs were smoothed with a Gaussian kernel of $10 \mathrm{~ms}$.

vidual examples of such cross-correlograms (CCs) obtained in four different animals are shown in Figure 1B1-B4. However, at odds with the idea that mPFC firing inhibited principal BLA neurons, the CCs suggested that mPFC firing was generally associated with an increased BLA firing probability that peaked $20-40 \mathrm{~ms}$ after $\mathrm{mPFC}$ firing.

To assess whether individual CCs were statistically significant, we compared each $10 \mathrm{~ms}$ bin in the $\pm 500 \mathrm{~ms}$ range to a baseline Poisson distribution of bin values, using a $0.05 p$ value corrected for multiple comparisons (Fig. 1). Overall, the CCs of 163 mPFC-BL cell couples (27.7\%) were deemed significant, or 5.5 times more than expected by chance $(p<0.01$; Fisher's exact test). Of these, 160 CCs showed a positive correlation. Sorting significant CCs by their peak time (Fig. 1C) revealed that 119 CCs (or 73\%) peaked after the mPFC reference (Fig. 1C, below horizontal black line) compared with only 44 (or $27 \%$ ) before (Fig. $1 C$, above horizontal line), which constitutes a significant imbalance $\left(p<0.01 ; \chi^{2}\right)$. This result was also evident in the average of all significant CCs (Fig. 1D1) in which an increased BLA activity was observed after the $\mathrm{mPFC}$ reference spike, peaking at $\sim 20-30$ ms (Fig. 1D1, expanded inset). The same phenomenon can be seen in the average of all 587 couples (Fig. 1D2). Consistent with this, the distribution of peak times (Fig. $1 E$, red line) was again unbalanced toward positive values with a clear maximum at the $0-50 \mathrm{~ms}$ bin. In contrast, the distribution of trough times (Fig. $1 E$, blue line) was homogenous in this range.

Together, these results indicate that mPFC firing is generally followed by an increased firing probability in BLA neurons, suggesting that the mPFC sends excitatory projections to the BLA.

\section{Effect of electrical mPFC stimulation on BLA neurons}

The above analyses imply that mPFC axons excite rather than inhibit BLA cells. If this were the case, one would expect electrical mPFC stimulation to evoke orthodromic discharges in most BLA cells. To test this point, we studied the neuronal responses in the BL nucleus to electrical stimulation of the mPFC. Because mPFC projections to the BLA nucleus arise from layer IIIII neurons (Russchen, 1982) (for review, see McDonald, 1998), we aimed our stimulating electrodes to superficial mPFClayers. To this end, we monitored spontaneous EEG activity (Fig. 2A1) through a concentric stimulating electrode $(0.7 \mathrm{~mm}$ spacing between tip and ring) as it was lowered toward the $\mathrm{MPFC}$ (Fig. 2A2). We advanced the electrode until the polarity of slow EEG events recorded by the tip became opposite to that recorded by the ring (Fig. 2A1) (see Materials and Methods). Histological controls consistently revealed that, in this position, the tip of the stimulating electrode was in layer I (Fig. 2A2).

The effect of mPFC stimuli was tested in 283 neurons histologically determined to be located in the BL nucleus. Of these, only 22 BL cells (or 7.8\%) were activated synaptically by mPFC stimuli and at long latencies $(37.0 \pm 2.1 \mathrm{~ms})$. In fact, the example of Figure $2 B$ is one of the shortest latency BL responses we observed after mPFC stimulation. Also unexpected was the fact that $\mathrm{mPFC}$ stimuli generally silenced spontaneously active BL cells, even when the tested cells were otherwise unresponsive (Fig. $2 C)$. In a subset of BLA cells, selected because they fired spontaneously at $2 \mathrm{~Hz}$ or more $(n=19), 95 \%$ were almost completely silenced for periods ranging between 100 and $500 \mathrm{~ms}$. An example of this is shown for a group of BL cells that were simultaneously recorded by the same microelectrode (Fig. 2C). Note that this should result in a disfacilitation, or in other words, remove a tonic excitatory input to the targets of BL neurons.

To further test the influence of mPFC inputs on BLA neuronal excitability, we examined the effect of mPFC shocks on the synaptic responsiveness of BL cells to other cortical inputs $(n=7)$ (Fig. 2D). To this end, conditioning mPFC stimuli (typically, a $0.05-0.2 \mathrm{~ms}$ pulse of $0.1-0.5 \mathrm{~mA}$ ) that did not evoke orthodromic discharges in the tested cell were delivered before a single test stimulus to the insula. The interstimulus interval (ISI) was varied from 10 to $90 \mathrm{~ms}$ and at least 20 stimuli were delivered at each ISI. As shown in Figure 2D, conditioning mPFC shocks caused a profound reduction $(66.5 \pm 15.2 \%)$ in the orthodromic responsiveness of $\mathrm{BL}$ cells to insula stimuli that peaked with $10-30$ ms ISIs.

\section{Comparison of $\mathrm{MPFC}$ and $\mathrm{BL}$ axon conduction times}

The results of the above two sets of experiments seem contradictory. The correlation of spontaneous MPFC and BLA discharges 
suggests that the mPFC sends strong excitatory projections to the BLA (Fig. 1). Yet electrical stimulation of the mPFC excites a low proportion of BLA cells and often has inhibitory effects on their spontaneous activity and synaptic responsiveness (Fig. 2). The contradiction, however, might be only apparent. Because the mPFC and BLA are reciprocally connected (for review, see McDonald, 1998), electrical stimulation of the mPFC should not only recruit $\mathrm{MPFC}$ axons that reach the BLA but also antidromically activate BLA axons that end in the mPFC. Because BLA projection cells terminating in the MPFC have local axon collaterals in the amygdala (McDonald, 1992; Smith et al., 2000), antidromic spikes might recruit local GABAergic interneurons and cause an artifactual inhibition of principal cells (Samson and Paré, 2003). Consistent with this possibility, we observed that as many as $32 \%$ of BLA cells (or 91 of 278 neurons) responded antidromically to $\mathrm{mPFC}$ stimuli. Antidromic spikes could be easily distinguished from orthodromic discharges because the former occurred at a fixed latency (Fig. 3A1) and collided with spontaneous action potentials (Fig. 3A2).

However, in order for backfired BL axons to trigger feedback inhibition before the recruitment of feedforward inhibition by $\mathrm{mPFC}$ inputs, the conduction times of $\mathrm{BL}$ axons to the mPFC should be shorter than that of mPFC axons ending in the amygdala. To test this idea, we directly compared the conduction times of $\mathrm{BL}$ (Fig. 4A1,A2) and mPFC axons (Fig. $4 B 1, B 2)$ using antidromic response latencies. In support of the feedback inhibition hypothesis, the antidromic responses of BL neurons to $\mathrm{mPFC}$ stimuli generally oc-

curred at shorter latencies (mode, $8 \mathrm{~ms}$; average, $21.9 \pm 1.2 \mathrm{~ms}$; $n=98$ ) (Fig. 4A2, thick line) than those seen in mPFC neurons in response to BL stimuli (mode, $24 \mathrm{~ms}$; average, $32.8 \pm 2.21 \mathrm{~ms}$; $n=27$; $t$ test; $t=4.25 ; \mathrm{df}=123 ; p<0.01$ ) (Fig. 4 B2, thick line). Figure 4 depicts examples of $\mathrm{mPFC}$ - and BL-evoked antidromic responses (dots) recorded in the $\mathrm{BL}$ nucleus (Fig. 4A1) and mPFC (Fig. 4B1), respectively. Using an estimated linear travel distance of $17 \mathrm{~mm}$ (Reinoso-Suárez, 1961) and the modes of our antidromic latency distributions (Fig. 4A2,B2, thick lines), we estimate that $\mathrm{BL}$ projection cells have a minimum conduction velocity of $2.1 \mathrm{~m} / \mathrm{s}$ compared with $0.70 \mathrm{~m} / \mathrm{s}$ for mPFC axons. The longer conduction times of $\mathrm{mPFC}$ axons may result from their smaller diameter and/or their longer path to the amygdala. In any event, the fact that $\mathrm{BL}$ axons to the $\mathrm{mPFC}$ have a shorter conduction time than $\mathrm{MPFC}$ axons to the amygdala allows for the possibility that the antidromic recruitment of BLA axons triggers feedback inhibition before the arrival of orthodromic mPFC impulses.

Consistent with the above analysis of antidromic response latencies, $\mathrm{mPFC}$-evoked orthodromic spikes in BL neurons generally occurred at a longer latency $(37.0 \pm 2.1 \mathrm{~ms}$; mode, $40 \mathrm{~ms}$; sulcus. Error bars indicate SE.
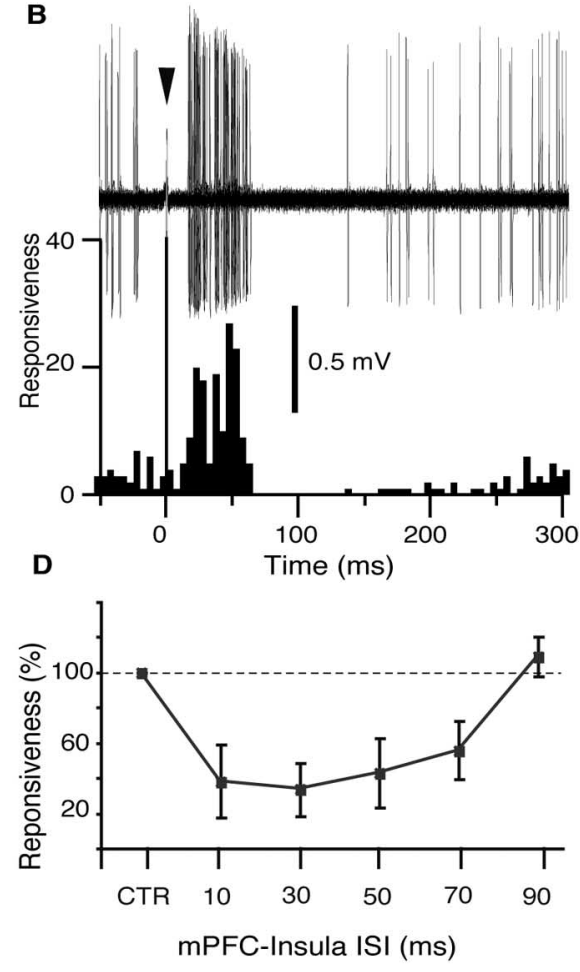

ime (s)
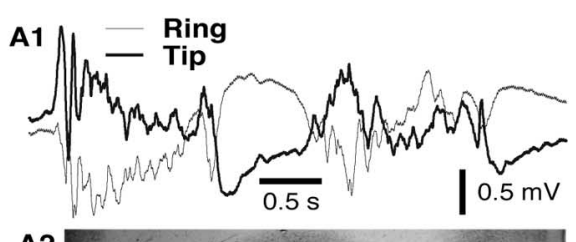

Figure 2. Effect of electrical mPFC stimulation on neuronal activity in the BL nucleus. $\mathbf{A 1}, \boldsymbol{A 2}$, Electrophysiological criteria used to adjust the position of a concentric stimulating electrode in superficial mPFC layers. A1, Electrode position was adjusted such that the EEG activity recorded by the tip (thick line) and ring (thin line) of the electrode ( $0.7 \mathrm{~mm}$ spacing) was of opposite polarity. In stand for dorsal, ventral, lateral, and medial, respectively. $\boldsymbol{B}$, Example of BL neuron orthodromically activated by mPFC stimulation

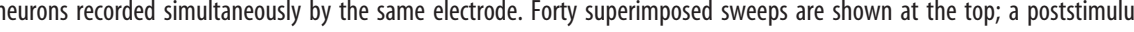
( 50 ms bins) based on 54 such sweeps is shown below using the same time base. The dashed line indicates average bin control) or preceded by mPFC stimuli with various ISIs ( $x$-axis). Data were obtained in four different animals. PSS, Presylvian

$n=22 ; t$ test; $t=5.43 ; \mathrm{df}=41 ; p<0.01$ ) (Fig. $4 A 2$, thin line) than those evoked in $\mathrm{mPFC}$ neurons after BL stimuli $(18.3 \pm 2.7$ ms; mode, $10 \mathrm{~ms} ; n=21$ ) (Fig. $4 B 2$, thin line). The distribution of antidromic and orthodromic response latencies to $\mathrm{MPFC}$ and BL stimuli are superimposed in Figure 4 (right panels).

mPFC stimulation compared with other cortical sites: effects in the $\mathrm{BL}$

If the low proportion of orthodromically activated BLA cells seen in response to $\mathrm{MPFC}$ stimulation is attributable to the antidromic invasion of BLA cells that subsequently recruit feedback interneurons, then it should be possible to reproduce this phenomenon by stimulating other cortical sites that have reciprocal connections with the BLA. In contrast, a higher proportion of orthodromically activated BLA cells should be seen when stimulating cortical inputs that project to the BLA without receiving return BLA inputs.

To test this idea, we compared $\mathrm{BL}$ responsiveness to stimulation of the insula, another cortical area with which the BL is reciprocally connected, versus the temporal neocortex, which innervates the BL without receiving return projections (Krettek and 

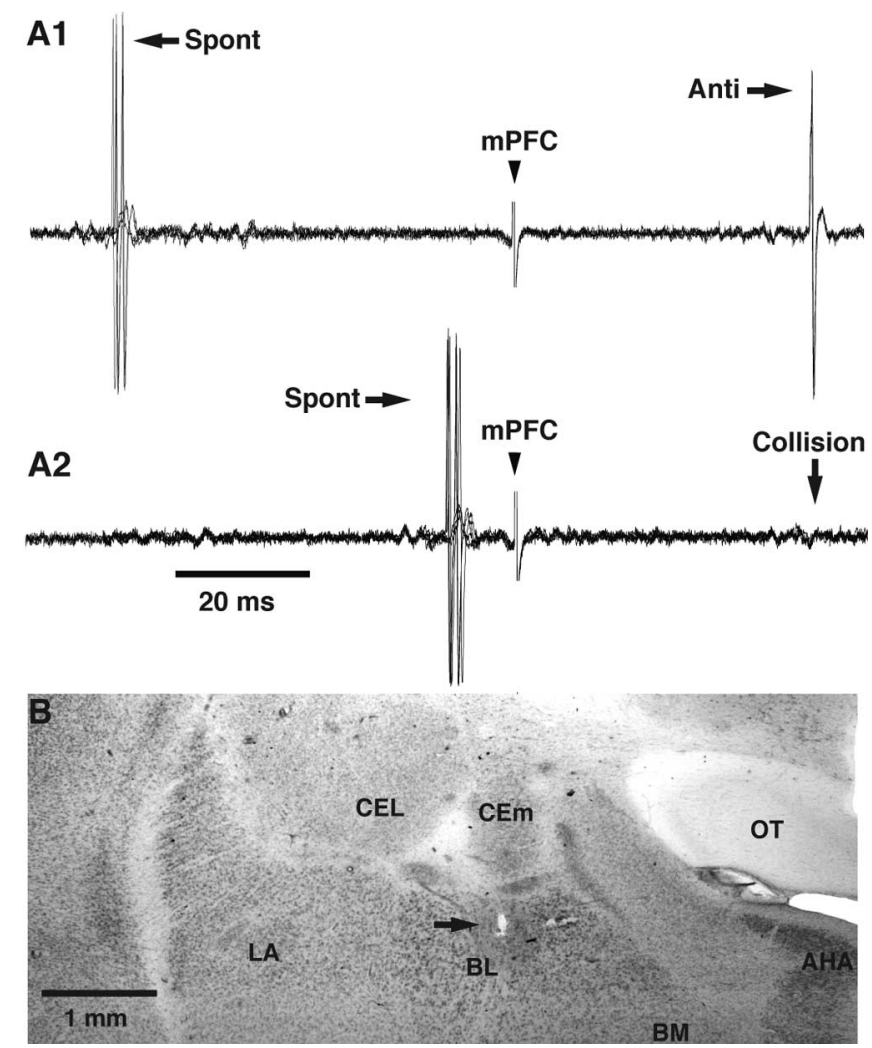

Figure 3. Physiological and histological identification of BL recording sites. $\boldsymbol{A} \mathbf{1}, \boldsymbol{A 2}$, Collision test used to determine whether an evoked spike was elicited by antidromic invasion. $\boldsymbol{A}$ 1, mPFC stimuli evoke antidromic spikes (Anti) at a fixed latency (three superimposed sweeps). A2, Spontaneous spikes (Spont) occurring within the collision interval abolish (Collision) the antidromic response. Collision does not occur when the interval between the spontaneous and antidromic spikes is outside the collision interval as in $\boldsymbol{A 1}$. $\boldsymbol{B}$, Coronal section of the amygdala stained with neutral red. The arrow points to an electrolytic lesion that marks the position of the first BL neuron antidromically responsive to $\mathrm{mPFC}$ stimuli encountered during the electrode track. This point coincides with the dorsal border of the BL nucleus, as predicted from tracttracing studies. AHA, Amygdalohippocampal area; BM, basomedial nucleus; CEL, central lateral nucleus; LA, lateral nucleus; $0 \mathrm{~T}$, optic tract.

Price, 1977; Russchen, 1982) (for review, see McDonald, 1998). The responsiveness of 283 BLA cells was tested (Fig. 5). As mentioned above, 32.2 and $7.8 \%$ of cells responded antidromically versus orthodromically to mPFC stimulation, respectively. Like mPFC shocks, insula stimulation elicited more antidromic (34.3\%; 97 of 283$)$ than orthodromic $(7.4 \% ; 21$ of 283$)$ responses (Fig. 5). Note that $27 \%$ (6 of 22 ) and $33 \%$ (7 of 21 ) of BL neurons, orthodromically activated from the mPFC and insula, respectively, could be backfired from one or more other cortical sites, indicating that they were projection cells. These results suggest that the response profile of $\mathrm{BL}$ neurons to $\mathrm{mPFC}$ stimuli is not unique to this cortical region but is shared by at least one other cortical area with which the $\mathrm{BL}$ is reciprocally connected: the insula.

Next, we examined the effects of electrical stimuli delivered in the temporal neocortex immediately lateral to perirhinal area 36 . Hereafter, this region will be called "area temp" for simplicity. Area temp receives very few inputs from the $\mathrm{BL}$ and sends a weaker projection to the $\mathrm{BL}$ than either the insula or the mPFC (Krettek and Price, 1977; Russchen, 1982). In support of this anatomical evidence, electrical stimulation of area temp elicited antidromic responses in a significantly lower proportion of BL neurons than the other two sites ( 41 cells or $14.5 \% ; \chi^{2}=24.769$;
A1 A2

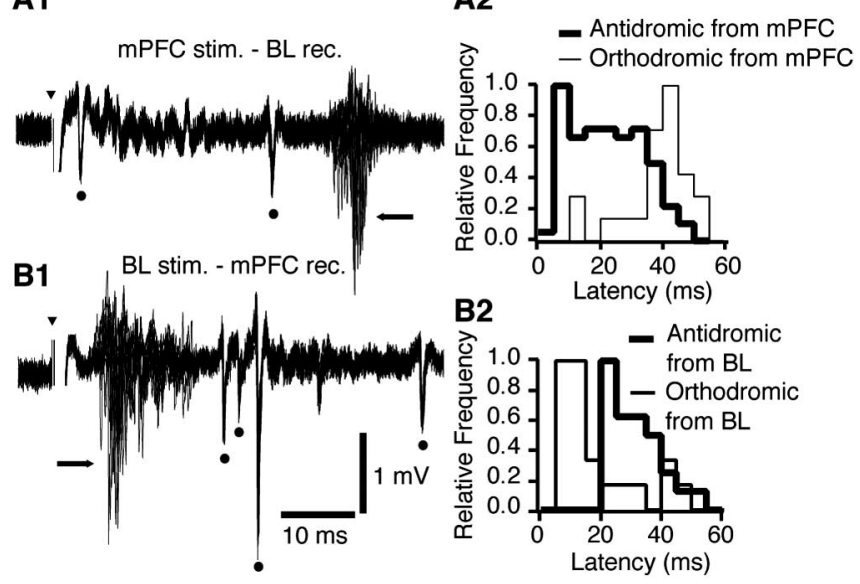

Figure 4. The axons of BL neurons projecting to the $\mathrm{MPFC}$ have a shorter conduction time than $\mathrm{mPFC}$ axons ending in the BL nucleus. $\boldsymbol{A}$ 1, Example of multiunit responses elicited by $\mathrm{mPFC}$ stimuli in the BL nucleus (20 superimposed sweeps). A2, Distribution of antidromic (thick lines) and orthodromic (thin lines) response latencies (all experiments combined). Each distribution was normalized to its mode. Antidromic response latencies ranged widely from 4 to $46 \mathrm{~ms}$, with a mode of $8 \mathrm{~ms}$. B1, Example of responses elicited by BL stimuli in the $\mathrm{MPFC}$ (20 superimposed sweeps). B2, Distribution of antidromic (thick lines) and orthodromic (thin lines) response latencies (all experiments combined). Antidromic response latencies ranged from 21 to $47 \mathrm{~ms}$, with a mode of $24 \mathrm{~ms}$. Dots and arrows mark antidromic and orthodromic responses, respectively. Bins of $5 \mathrm{~ms}$ in $\boldsymbol{A} \mathbf{2}$ and $\boldsymbol{B} \mathbf{2}$. Arrowheads indicate stimulation artifacts.

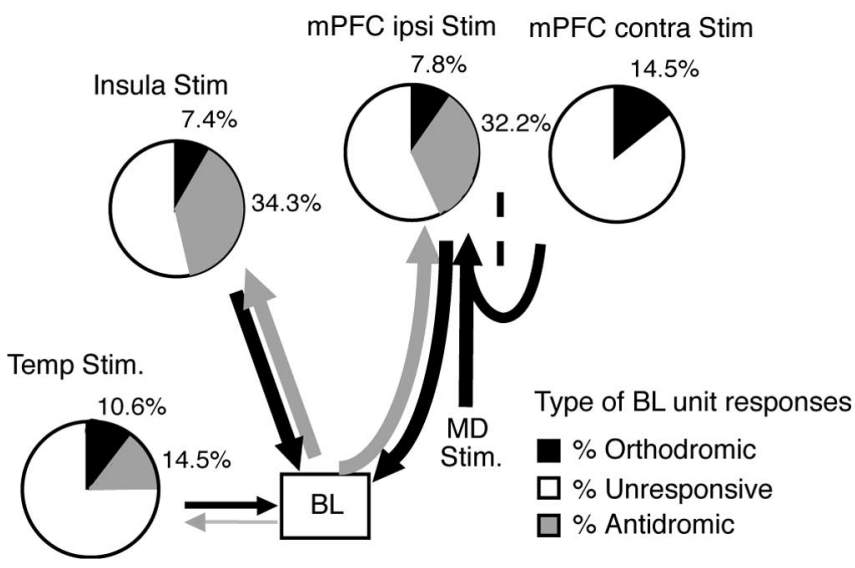

Figure 5. Depending on the strength and directionality of connections, electrical stimulation of different cortical areas elicits contrasting patterns of BL unit responsiveness. Pie charts show relative incidence of orthodromically responsive (black), antidromically responsive (gray), and unresponsive BL cells with various stimulation sites (area temp, bottom left; insula, top left; ipsilateral $\mathrm{mPFC}$, center; indirect activation of the $\mathrm{MPFC}$, top right). Thickness of arrows indicates relative strength of connections between stimulation sites and BL nucleus, as described in previous tract-tracing studies (see references in Results). Temp, Temporal neocortex; Stim, stimulation; ipsi, ipsilateral; contra, contralateral.

$\mathrm{df}=2 ; p<0.01)$. Yet, despite a weaker projection of this cortical area to the BL, as many as $10.6 \%$ (or 30 of 283) of the tested cells were orthodromically activated after stimulation of area temp (Fig. 5).

mPFC-mediated synaptic excitation of BL pyramidal cells Thus far, we have argued that electrical mPFC stimuli backfire a proportion of BL projection cells, thus causing widespread feedback inhibition in the BL nucleus and reducing the number of cells that are orthodromically activated by mPFC inputs. To establish this point directly, we then compared the proportion of orthodromically activated BL cells observed after direct mPFC 
stimulation versus stimuli that activate the mPFC indirectly, thus eliminating the confound of antidromic BL responses. To activate the mPFC indirectly, we stimulated the contralateral $\mathrm{mPFC}$ and the MD. The contralateral mPFC projects to the ipsilateral $\mathrm{mPFC}$ via the corpus callosum (Vertes, 2004), whereas the MD sends a large projection to the ipsilateral $\mathrm{mPFC}$ (Musil and Olson, 1991). Importantly, neither the MD nor the contralateral $\mathrm{mPFC}$ receive $\mathrm{BLA}$ projections in cats (Krettek and Price, 1977).

As mentioned above, direct electrical stimulation of the ipsilateral mPFC evoked orthodromic spikes in $7.8 \%$ of recorded BL cells (22 of 283). However, when the mPFC was activated indirectly, by stimulating the contralateral mPFC or ipsilateral $\mathrm{MD}$, a significantly higher proportion of BL cells was orthodromically activated (14.5\%; 37 of 256) (Fig. 5). The differences in the proportion of orthodromically responsive cells in the $\mathrm{BL}\left(\chi^{2}=3.81 ; \mathrm{df}=1\right.$; $p<0.05)$ indicate that direct electrical stimulation of the ipsilateral $\mathrm{mPFC}$ may result in an artifactual bias toward inhibition.

To confirm that MD stimulation activated $B L$ neurons by way of the $\mathrm{mPFC}$, we examined whether inactivation of the ipsilateral mPFC with lidocaine could block MD-evoked orthodromic activation of physiologically identified BL projection cells (Fig. 6). After finding a suitable BLA projection neuron and documenting its evoked activity, lidocaine was slowly infused in the $\mathrm{mPFC}(0.2 \mu \mathrm{l} / \mathrm{min} ; 20-40$ $\min ; n=5)$. During the lidocaine infusion, the spontaneous and evoked activity of the tested cells was monitored at 5 min intervals. In five of five tested cells, lidocaine infusion in the mPFC abolished MDevoked responses without affecting their spontaneous firing rate ( $t$ test; $p>0.05$ ) within 20-40 min. Figure 6 shows a representative example of such an experiment. This BL neuron was antidromically responsive to electrical stimulation of the ipsilateral mPFC (Fig. 6A1, dot) and orthodromically activated by electrical stimuli delivered in the MD (Fig. 6B1) or area temp region (Fig. $6 C 1$ ). MD-evoked unit responses were completely abolished after lidocaine infusion (Fig. 6 B2), as were MD-evoked field potentials in the $\mathrm{mPFC}$ (Fig. 6A2). This was not caused by lidocaine diffusion to the amygdala or recording instability, because the response evoked by stimulation of area temp persisted and the spike shape remained stable (Fig. 6C2).

\section{Discussion}

This study was undertaken to determine how the mPFC affects the amygdala. The importance of this issue derives from functional imaging evidence of reduced MPFC activity in PTSD subjects (Bremner et al., 1999; Shin et al., 2004) and data implicating $\mathrm{mPFC}$-amygdala interactions in the extinction of conditioned fear. In particular, protein synthesis inhibition in the MPFC (Santini et al., 2004) and mPFC lesions (Morgan et al., 1993; Quirk et
A2

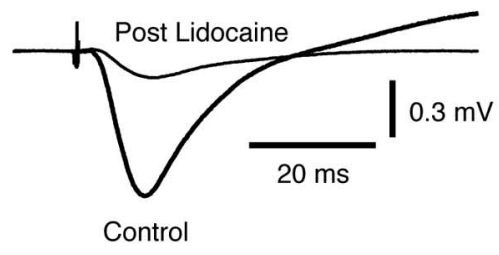

B2 MD 40 min Post Lidocaine
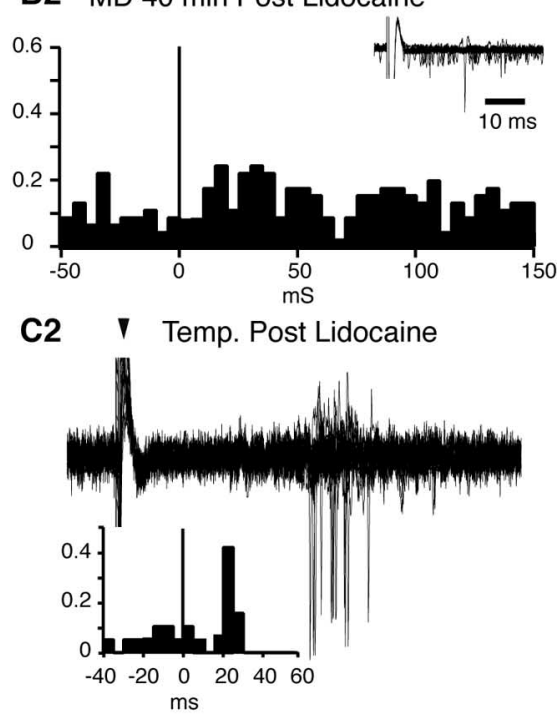

Figure 6. Lidocaine infusion in the $\mathrm{mPFC}$ blocks an orthodromic response of a BL projection cell evoked by MD stimulation. $A B L$ Shown. Lidocaine infusion in the MPFC reduced MD-evoked field potentials in the MPFC (A2) and orthodromic spiking of the tested BL neuron (B2). In contrast, orthodromic responsiveness of the tested cell to stimulation of area temp was stimuli. Insets show 20 superimposed sweeps. Poststimulus histograms in $\mathbf{C} 1$ and $\mathbf{C} 2$ were constructed from responses to 40 area temp stimuli. Fifteen superimposed sweeps are depicted. In $\mathbf{B} \mathbf{1}, \mathbf{B} \mathbf{2}, \mathbf{C}$, and $\mathbf{C}$, we depict only sweeps devoid of spontaneous firing in the $100 \mathrm{~ms}$ preceding the stimuli. Arrowheads indicate stimulation artifacts.

al., 2000) disrupt extinction recall. Moreover, mPFC neurons exhibit CS-evoked responses during extinction recall (Milad and Quirk, 2002), and mPFC stimulation reduces conditioned freezing (Milad and Quirk, 2002) and inhibits CEm output neurons (Quirk et al., 2003).

However, the latter finding is in contradiction to anatomical data predicting that $\mathrm{mPFC}$ inputs should activate BLA neurons and thus excite BLA projection sites such as the CEm (Krettek and Price, 1978; Paré et al., 1995; Pitkänen et al., 1997). This led us to test the possibility that MPFC inputs recruit GABAergic interneurons of the BLA, inhibiting BLA projection cells and causing a disfacilitation of CEm neurons. At odds with this scenario, however, we found that $\mathrm{mPFC}$ inputs excite rather than inhibit BLA neurons.

mPFC activity is correlated with an increase in the firing probability of BLA neurons

We observed a positive correlation between the spontaneous discharges of simultaneously recorded $\mathrm{mPFC}$ and BLA neurons. Although we cannot rule out the possibility that this correlation is a consequence of a common excitatory input that reaches the $\mathrm{mPFC}$ and BLA with a time lag, several factors support the inter- 
pretation that it results from the strong excitatory projections sent by the mPFC to the BLA.

Most importantly, the average delay seen between MPFC and BLA firing in the cross-correlograms matches the conduction time of mPFC axons to the amygdala, as estimated using antidromic response latencies. Second, $\mathrm{mPFC}$ axon terminals form only asymmetric synapses in the BLA, a type of synaptic junction usually formed by excitatory axons (Colonnier,1981). Third, $>90 \%$ of the synapses formed by mPFC axons in the BLA are found on spines. Because BLA interneurons are aspiny or sparsely spiny, the main targets of $\mathrm{mPFC}$ axons are likely projection neurons rather than interneurons.

\section{Direct electrical stimulation of the mPFC elicits an artifactual inhibition of BLA projection cells}

At odds with anatomical data suggesting that $\mathrm{MPFC}$ inputs should exert excitatory effects in the BLA, electrical stimulation of the mPFC activated a low proportion of BL neurons synaptically, silenced most spontaneously active BL cells, and generally reduced the synaptic responsiveness of BL cells to other cortical inputs. This apparent contradiction, as well as the positive correlation seen between $\mathrm{mPFC}$ and BLA firing, led us to investigate the possibility that electrical $\mathrm{MPFC}$ stimulation causes an artifactual inhibition of the BLA, because such stimuli backfire a proportion of BLA projection cells, which, via their local axon collaterals, recruit feedback interneurons.

To test this idea, we compared the conduction time of mPFC versus $\mathrm{BL}$ axons and found that $\mathrm{BL}$ axons terminating in the mPFC have much shorter conduction times than mPFC axons ending in the BL. This allows for the possibility that mPFC stimulation recruits $\mathrm{BL}$ interneurons, before the arrival of $\mathrm{MPFC}$ impulses to the BL. In a second test, we compared the pattern of BL responsiveness to electrical stimulation of various cortical sites and found that the likelihood of eliciting orthodromic spikes in the $\mathrm{BL}$ is not proportional to the strength of the cortical projection but is lower for cortical areas that have strong reciprocal connections with the BLA. In particular, we found that electrical stimulation of the mPFC or insula, both of which have strong reciprocal connections with the BL nucleus (Shi and Cassell, 1998) (for review, see McDonald, 1998), elicited more antidromic than orthodromic responses in BL neurons. In contrast, area temp stimulation, which sends fewer projections to the $\mathrm{BL}$ than the insula or $\mathrm{mPFC}$ but receives few return projections from the BL (Krettek and Price, 1977; Russchen, 1982), synaptically excited a higher proportion of BL neurons than either $\mathrm{MPFC}$ or insula stimuli. Given that area temp sends a much weaker projection to BL than the mPFC or insula, its comparable effectiveness in orthodromically activating BL neurons could mean that area temp synapses have a larger impact on BLA neurons than those contributed by the mPFC or insula. However, given that cortical axon terminals in the amygdala have uniform ultrastructural features regardless of their origin (Smith et al., 2000), a more parsimonious interpretation is that area temp stimuli elicit more orthodromic responses because they evoke less feedback inhibition. Consistent with this, indirect mPFC stimulation (by activating its thalamic or cortical afferents) unmasked excitation of physiologically identified BLA projection cells, presumably by eliminating the artifactual inhibition of BL neurons that resulted from the antidromic recruitment of local interneurons.

These conclusions are at odds with a previous study in which it was reported that electrical $\mathrm{mPFC}$ stimuli inhibit BLA projection cells in rats (Rosenkranz and Grace, 2001). These authors proposed that the inhibition of BLA projection cells resulted from the fact that $\mathrm{MPFC}$ inputs prevalently excite feedforward interneurons of the BLA. The possibility that mPFC stimulation triggered artifactual feedback inhibition of BLA projection cells was considered unlikely, because their mPFC stimuli were thought to be too brief to evoke antidromic discharges (Rosenkranz and Grace, 2001). Although it is possible that methodological (e.g., anesthetics) or species differences account for the discrepancies between these studies, it seems unlikely that direct electrical stimulation of the mPFC could selectively excite mPFC soma and dendrites as opposed to BLA axons. Indeed, antidromic responses can be elicited with a wide range of stimulation intensities (Lipski, 1981), depending on the distance between the activated axon and the stimulating electrodes (Ranck, 1975). Moreover, this interpretation cannot account for the positive correlation seen between the spontaneous discharges of $\mathrm{mPFC}$ and BLA neurons observed here.

\section{Extinction and the mPFC-amygdala circuit}

We showed that mPFC shocks evoke a mixture of orthodromic and antidromic discharges in the BLA. However, antidromic discharges occur first because BLA axons to the $\mathrm{mPFC}$ have shorter conduction times than mPFC axons to the BLA. Thus, the initial effect of electrical mPFC stimuli should be to activate the axon collaterals of antidromically invaded BLA neurons. Because BLA neurons send glutamatergic projections to the CEm (Smith and Paré, 1994; Paré et al., 1995), the antidromic activation of BLA neurons should cause orthodromic excitation of CEm cells. However, mPFC stimulation does not excite CEm cells (Quirk et al., 2003). These findings imply that the CEm is not innervated by the BLA neurons that also project to the $\mathrm{MPFC}$, but by a different subset of BLA cells that is likely inhibited after mPFC stimulation. Thus, from the standpoint of the CEm, the main effect exerted by mPFC shocks via the BLA is a disfacilitation. This raises the possibility that the mPFC-evoked inhibition of CEm cells might itself be an artifact of electrical stimulation.

However, several lines of evidence suggest that it is not the case. First, in the study by Quirk et al. (2003), mPFC shocks could even abolish the orthodromic spiking evoked in CEm cells by direct electrical stimulation of the BLA. Because such stimuli should have overwhelmed the mPFC-evoked BLA inhibition, these observations imply that mPFC stimuli inhibited the CEm via a gating mechanism downstream of the BLA. Second, in addition to innervating the BLA, the $\mathrm{mPFC}$ sends strong projections to the GABAergic intercalated cell masses (McDonald et al., 1996), which in turn project to the CEm (Paré and Smith, 1993; Royer et al., 1999). Indeed, it was recently reported that disinhibition of the $\mathrm{MPFC}$ with picrotoxin produces a large increase in the number of Fos-immunoreactive intercalated neurons (Berretta et al., 2005).

These findings lead us to hypothesize that the mPFC inhibits CEm cells by activating one or more populations of GABAergic cells, such as intercalated neurons, that receive direct $\mathrm{mPFC}$ inputs and project to the CEm (Paré and Smith, 1993; Royer et al., 1999; Pinto and Sesack, 2002). Although the mPFC also projects to the bed nucleus of the stria terminalis (Vertes, 2004), the possibility that intercalated neurons are involved is particularly appealing because BLA inputs to intercalated cells express activityand NMDA-dependent long-term potentiation (Royer and Paré, 2002, 2003). This finding, coupled with the sensitivity of extinction to intra-amygdaloid injections of NMDA receptor antagonists (Falls et al., 1992), raises the possibility that the acquisition of CS-evoked responses by $\mathrm{mPFC}$ neurons after extinction serves to bring about sufficient depolarization of intercalated cells for 
the NMDA-dependent potentiation of BLA inputs. As a result, during subsequent CS presentations, BLA cells would evoke relatively more inhibition than excitation in CEm output neurons, eventually leading to the suppression of conditioned fear responses.

The possibility that intercalated cells are critical for the extinction of conditioned fear might open new pharmacological avenues for the treatment of anxiety disorders. For instance, intercalated cells express a high concentration of $\mu$-opioid (Mansour et al., 1995; Wilson et al., 2002) and dopamine type 1 receptors, whereas $\mathrm{mPFC}$ inputs to intercalated cells express dopamine type 2 receptors (Maltais et al., 2000; Pinto and Sesack, 2002; Fuxe et al., 2003). Such data raise the prospect of targeted pharmacological interventions for accelerating extinction.

\section{References}

Bechara A, Tranel D, Damasio H, Adolphs R, Rockland C, Damasio AR (1995) Double dissociation of conditioning and declarative knowledge relative to the amygdala and hippocampus in humans. Science 269:1115-1118

Berretta S, Pantazopoulos P, Pantazopoulos H, Paré D (2005) Infralimbic cortex activation increases c-Fos expression in intercalated neurons of the amygdala. Neuroscience 132:943-953.

Boehnke SE, Rasmusson DD (2001) Time course and effective spread of lidocaine and tetrodotoxin delivered via microdialysis: an electrophysiological study in cerebral cortex. J Neurosci Methods 105:133-141.

Bremner JD, Staib LH, Kaloupek D, Southwick SM, Soufer R, Charney DS (1999) Neural correlates of exposure to traumatic pictures and sound in Vietnam combat veterans with and without posttraumatic stress disorder: a positron emission tomography study. Biol Psychiatry 45:806-816.

Brinley-Reed M, Mascagni F, McDonald AJ (1995) Synaptology of prefrontal projections to the basolateral amygdala: an electron microscopic study in the rat. Neurosci Lett 202:45-48.

Collins DR, Pelletier JG, Paré D (2001) Slow and fast (gamma) neuronal oscillations in the perirhinal cortex and lateral amygdala. J Neurophysiol 85:1661-1672.

Colonnier M (1981) The electron-microscopic analysis of the neuronal organization of the cerebral cortex. In: The organization of the cerebral cortex (Schmitt FO, Worden FG, Adelman G, Dennis SG, eds), pp 125152. Cambridge, MA: MIT.

Falls WA, Miserendino MJD, Davis M (1992) Extinction of fear-potentiated startle: blockade by infusion of an NMDA antagonist into the amygdala. J Neurosci 12:854-863.

Fuxe K, Jacobsen KX, Hoistad M, Tinner B, Jansson A, Staines WA, Agnati LF (2003) The dopamine D1 receptor-rich main and paracapsular intercalated nerve cell groups of the rat amygdala: relationship to the dopamine innervation. Neuroscience 119:733-746.

Gabbott PL, Bacon SJ (1996) Local circuit neurons in the medial prefrontal cortex (areas 24a,b,c, 25 and 32) in the monkey: II. Quantitative areal and laminar distributions. J Comp Neurol 364:609-636.

Hobin JA, Goosens KA, Maren S (2003) Context-dependent neuronal activity in the lateral amygdala represents fear memories after extinction. J Neurosci 23:8410-8416.

Holstege G, Bandler R, Saper CB (1996) The emotional motor system. Prog Brain Res 107:3-6.

Krettek JE, Price JL (1977) Projections from the amygdaloid complex to the cerebral cortex and thalamus in the rat and cat. J Comp Neurol 172:687-722.

Krettek JE, Price JL (1978) A description of the amygdaloid complex in the rat and cat with observations on intra-amygdaloid axonal connections. J Comp Neurol 178:255-280.

LaBar KS, LeDoux JE, Spencer DD, Phelps EA (1995) Impaired fear conditioning following unilateral temporal lobectomy in humans. J Neurosci 15:6846-6855.

LeDoux JE (2000) Emotion circuits in the brain. Annu Rev Neurosci 23:155-184.

Lipski J (1981) Antidromic activation of neurones as an analytic tool in the study of the central nervous system. J Neurosci Methods 4:1-32.

Maltais S, Coté S, Drolet G, Falardeau P (2000) Cellular colocalization of dopamine D1 mRNA and D2 receptor in rat brain using a dopamine receptor specific polyclonal antibody. Prog Neuropsychopharmacol Biol Psychiatry 24:1127-1149.

Mansour A, Fox CA, Burke S, Akil H, Watson JJ (1995) Immunohistochemical localization of the cloned mu-opioid receptor in the rat CNS. J Chem Neuroanatomy 8:283-305.

Maren S (2001) Neurobiology of Pavlovian fear conditioning. Annu Rev Neurosci 24:897-931.

Martinez-Moreno E, Llamas A, Avendano C, Renes E, Reinoso-Suarez F (1987) General plan of the thalamic projections to the prefrontal cortex in the cat. Brain Res 407:17-26.

McDonald AJ (1991) Organization of amygdaloid projections to the prefrontal cortex and associated striatum in the rat. Neuroscience 44:1-14.

McDonald AJ (1992) Cell types and intrinsic connections of the amygdala. In: The amygdala: neurobiological aspects of emotion, memory, and mental dysfunction (Aggleton JP, ed), pp 67-96. New York: Wiley.

McDonald AJ (1998) Cortical pathways to the mammalian amygdala. Prog Neurobiol 55:257-332.

McDonald AJ, Mascagni F (2001) Colocalization of calcium-binding proteins and GABA in neurons of the rat basolateral amygdala. Neuroscience 105:681-693.

McDonald AJ, Mascagni F, Guo L (1996) Projections of the medial and lateral prefrontal cortices to the amygdala: a Phaseolus vulgaris leucoagglutinin study in the rat. Neuroscience 71:55-75.

Milad MR, Quirk GJ (2002) Neurons in medial prefrontal cortex signal memory for fear extinction. Nature 420:70-74.

Morgan MA, LeDoux JE (1995) Differential contribution of dorsal and ventral medial prefrontal cortex to the acquisition and extinction of conditioned fear in rats. Behav Neurosci 109:681-688.

Morgan MA, Romanski LM, LeDoux JE (1993) Extinction of emotional learning: contribution of medial prefrontal cortex. Neurosci Lett 163:109-113

Musil SY, Olson CR (1991) Cortical areas in the medial frontal lobe of the cat delineated by quantitative analysis of thalamic afferents. J Comp Neurol 308:457-466.

Myers KM, Davis M (2002) Behavioral and neural analysis of extinction. Neuron 36:567-584.

Paré D, Smith Y (1993) The intercalated cell masses project to the central and medial nuclei of the amygdala in cats. Neuroscience 57:1077-1090.

Paré D, Smith Y, Paré JF (1995) Intra-amygdaloid projections of the basolateral and basomedial nuclei in the cat: Phaseolus vulgarisleucoagglutinin anterograde tracing at the light and electron microscopic level. Neuroscience 69:567-583.

Pavlov IP (1927) Conditioned reflexes: an investigation of the physiological activity of the cerebral cortex. London: Oxford UP.

Phelps EA, Delgado MR, Nearing KI, LeDoux JE (2004) Extinction learning in humans: role of the amygdala and vmPFC. Neuron 43:897-905.

Pinto A, Sesack SR (2002) Prefrontal cortex projection to the rat amygdala: ultrastructural relationship to dopamine $\mathrm{D}_{1}$ and $\mathrm{D}_{2}$ receptors. Soc Neurosci Abstr 28:587.6.

Pitkänen A, Savander V, LeDoux JE (1997) Organization of intraamygdaloid circuitries in the rat: an emerging framework for understanding functions of the amygdala. Trends Neurosci 20:517-523.

Quirk GJ, Repa JC, LeDoux JE (1995) Fear conditioning enhances shortlatency auditory responses of lateral amygdala neurons: parallel recordings in the freely behaving rat. Neuron 15:1029-1039.

Quirk GJ, Russo GK, Barron JL, Lebron K (2000) The role of ventromedial prefrontal cortex in the recovery of extinguished fear. J Neurosci 20:6225-6231.

Quirk GJ, Likhtik E, Pelletier JG, Paré D (2003) Stimulation of medial prefrontal cortex decreases the responsiveness of central amygdala output neurons. J Neurosci 23:8800-8807.

Ranck Jr JB (1975) Which elements are excited in electrical stimulation of mammalian central nervous system: a review. Brain Res 98:417-440.

Reinoso-Suárez F (1961) Topographischer hirnatlas der katze für experimental-physiologische untersuchungen. Darmstadt, Germany: E Merk AG.

Repa JC, Muller J, Apergis J, Desrochers TM, Zhou Y, LeDoux JE (2001) Two different lateral amygdala cell populations contribute to the initiation and storage of memory. Nat Neurosci 4:724-731.

Ressler KJ, Rothbaum BO, Tannenbaum L, Anderson P, Graap K, Zimand E, Hodges L, Davis M (2004) Cognitive enhancers as adjuncts to psycho- 
therapy: use of D-cycloserine in phobic individuals to facilitate extinction of fear. Arch Gen Psychiatry 61:1136-1144.

Rosenkranz JA, Grace AA (2001) Dopamine attenuates prefrontal cortical suppression of sensory inputs to the basolateral amygdala of rats. J Neurosci 21:4090-4103.

Royer S, Paré D (2002) Bidirectional synaptic plasticity in intercalated amygdala neurons and the extinction of conditioned fear responses. Neuroscience 115:455-462.

Royer S, Paré D (2003) Conservation of total synaptic weights via inverse homo- vs. heterosynaptic LTD and LTP. Nature 422:518-522.

Royer S, Martina M, Paré D (1999) An inhibitory interface gates impulse traffic between the input and output stations of the amygdala. J Neurosci 19:10575-10583.

Russchen FT (1982) Amygdalopetal projections in the cat. I. Cortical afferent connections. A study with retrograde and anterograde tracing techniques. J Comp Neurol 206:159-179.

Samson R, Paré D (2003) Feedback inhibition defines transverse processing modules in the lateral amygdala. J Neurosci 23:1966-1973.

Santini E, Ge H, Ren K, Pena de Ortiz S, Quirk GJ (2004) Consolidation of fear extinction requires protein synthesis in the medial prefrontal cortex. J Neurosci 24:5704-5710.

Sesack SR, Deutch AY, Roth RH, Bunney BS (1989) Topographical organization of the efferent projections of the medial prefrontal cortex in the rat: an anterograde tract-tracing study with Phaseolus vulgaris leucoagglutinin. J Comp Neurol 290:213-242.

Shi CJ, Cassell MD (1998) Cortical, thalamic, and amygdaloid connections of the anterior and posterior insular cortices. J Comp Neurol 399:440-468.

Shin LM, Orr SP, Carson MA, Rauch SL, Macklin ML, Lasko NB, Peters PM, Metzger LJ, Dougherty DD, Cannistraro PA, Alpert NM, Fischman AJ, Pitman RK (2004) Regional cerebral blood flow in the amygdala and medial prefrontal cortex during traumatic imagery in male and female Vietnam veterans with PTSD. Arch Gen Psychiatry 61:168-176.

Smith Y, Paré D (1994) Intra-amygdaloid projections of the lateral nucleus in the cat: PHA-L anterograde labeling combined with post-embedding GABA and glutamate immunocytochemistry. J Comp Neurol 342:232-248.

Smith Y, Paré JF, Paré D (2000) Differential innervation of parvalbuminimmunoreactive interneurons of the basolateral amygdaloid complex by cortical and intrinsic inputs. J Comp Neurol 416:496-508.

Steriade M, Nuñez A, Amzica F (1993) A novel slow ( $<1 \mathrm{~Hz}$ ) oscillation of neocortical neurons in vivo: depolarizing and hyperpolarizing components. J Neurosci 13:3252-3265.

Vertes RP (2004) Differential projections of the infralimbic and prelimbic cortex in the rat. Synapse 51:32-58.

Walker DL, Ressler KJ, Lu KT, Davis M (2002) Facilitation of conditioned fear extinction by systemic administration or intra-amygdala infusions of D-cycloserine as assessed with fear-potentiated startle in rats. J Neurosci 22:2343-2351.

Wilson MA, Mascagni F, McDonald AJ (2002) Sex differences in delta opioid receptor immunoreactivity in rat medial amygdala. Neurosci Lett 328:160-164. 